\title{
KEYNOTE-590: Phase III study of first-line chemotherapy with or without pembrolizumab for advanced esophageal cancer
}

\author{
Ken Kato*,1, Manish A Shah ${ }^{2}$, Peter Enzinger ${ }^{3}$, Jaafar Bennouna ${ }^{4}$, Lin Shen ${ }^{5}$, Antoine \\ Adenis ${ }^{6}$, Jong-Mu Sun ${ }^{7}$, Byoung Chul $\mathrm{Cho}^{8}{ }^{2}$ Mustafa Özgüroğlu ${ }^{9}$, Takashi Kojima ${ }^{10}$, \\ Vladimir Kostorov ${ }^{11}$, Cinta Hierro ${ }^{12}$, Ying Zhu ${ }^{13}$, Lee Anne McLean ${ }^{13}$, Sukrut Shah ${ }^{13}$ \& \\ Toshihiko Doi ${ }^{10}$ \\ ${ }^{1}$ National Cancer Center Hospital, 5-1-1 Tsukiji, Chuo-ku, Tokyo, 104-0045, Japan \\ ${ }^{2}$ Weill Cornell Medical College, New York Presbyterian Hospital, 1305 York Avenue, Room Y1247, New York, NY 10065, USA \\ ${ }^{3}$ Dana-Farber Cancer Institute, 450 Brookline Avenue, Boston, MA 02215-5450, USA \\ ${ }^{4}$ University Hospital of Nantes, Digestive Oncology, 1 Place Alexis Ricordeau, Nantes, 44000, France \\ ${ }^{5}$ Peking University Cancer Hospital \& Institute, 52 Fucheng Road, Haidian District, Beijing, 100142, PR China \\ ${ }^{6}$ Institut du Cancer de Montpellier, 208 Avenue des Apothicaries, Montpellier, 34298, France \\ ${ }^{7}$ Samsung Medical Center, Sungkyunkwan University School of Medicine, 81 Irwon-ro, Seoul, Republic of Korea \\ ${ }^{8}$ Severance Hospital, Yonsei University Health System, 50-1 Yonsei-ro, Seoul, Republic of Korea \\ ${ }^{9}$ Istanbul University-Cerrahpaşa, Cerrahpaşa School of Medicine, Koca Mustafa Paşa Mahallesi, Cerrahpaşa Caddesi No. 53, \\ Istanbul, 34096, Turkey \\ ${ }^{10}$ National Cancer Center Hospital East, 6-5-1 Kashiwanoha, Kashiwa, 277-8577, Japan \\ ${ }^{11}$ Leningrad Regional Oncology Dispensary, Ulitsa Savushkina, 126, Saint Petersburg, 197374, Russian Federation \\ ${ }^{12}$ Vall d'Hebron University Hospital \& Vall d'Hebron Institute of Oncology (VHIO), 119-129 Pg vall d'Hebron, Barcelona, 08035, \\ Spain \\ ${ }^{13}$ Merck \& Co., Inc., 2000 Galloping Hill Road, Kenilworth, NJ 07033, USA \\ *Author for correspondence: Tel.: +81 33542 2511; kenkato@ncc.go.jp
}

Background: Treatment options for patients with advanced esophageal or esophagogastric junction (EGJ) cancer are limited. Current guidelines for first-line treatment of advanced esophageal or EGJ cancer recommend chemotherapy containing a platinum and a fluoropyrimidine agent. Pembrolizumab demonstrated antitumor activity in previously treated patients with advanced esophageal cancer and in patients with gastroesophageal junction cancer. Aim: To describe the design and rationale for the randomized, double-blind, placebo-controlled Phase III KEYNOTE-590 study, which will be conducted to investigate pembrolizumab in combination with chemotherapy as first-line treatment in patients with advanced esophageal or EGJ cancer.

Clinical trial registry \& ID: ClinicalTrials.gov: NCT03189719.

First draft submitted: 10 August 2018; Accepted for publication: 20 December 2018; Published online: 8 February 2019

Keywords: chemotherapy $\bullet$ esophageal cancer $\bullet$ esophagogastric junction cancer $\bullet$ gastrointestinal/esophageal • immunotherapy • PD-1 • PD-L1 • pembrolizumab • squamous cell carcinoma of the esophagus

Esophageal cancer is the eighth most commonly diagnosed cancer worldwide and the sixth most common cause of cancer-related death (incidence, approximately 456,000; mortality, 400,000 in 2012) [1]. Incidence varies significantly between regions, with age-standardized rates ranging from 2.2 per 100,000 in the Middle East and Northern Africa to 10.2 per 100,000 in the Western Pacific region [2]. It is estimated that, in 2018, 17,000 people were diagnosed with esophageal cancer in the USA and almost 16,000 died of the disease [3]. The 5-year survival rate for patients with diagnosed esophageal cancer in the USA is only $18 \%$, with poor outcomes attributed to late diagnosis and a propensity for metastases $[4,5]$.

Future Medicine 
Most esophageal cancers can be categorized into two main histologic subtypes: squamous cell carcinoma (SCC) and adenocarcinoma. SCC is the most common esophageal cancer subtype diagnosed worldwide; however, there has been a shift in the past four decades toward an increasing incidence of adenocarcinoma in western populations [6,7].

Treatment options for patients with unresectable advanced or metastatic esophageal or esophagogastric junction (EGJ) cancer are limited. Current guidelines for the first-line treatment of advanced or metastatic disease recommend platinum-based chemotherapy in combination with fluoropyrimidine [8,9]. Although European Society for Medical Oncology guidelines recommend monotherapy or best supportive care as first-line therapy in patients with SCC [8], platinum doublet chemotherapy is commonly used without supportive evidence from randomized controlled trials in Asian countries, with response rates of only 20-50\% [10]. For patients whose disease progresses on first-line therapy, there is no consensus on a second-line regimen, although docetaxel, paclitaxel and irinotecan are commonly used for both histologic subtypes [11-16]. The effectiveness of targeted agents has also been investigated. Results of the Trastuzumab for Gastric Cancer trial demonstrated that trastuzumab plus chemotherapy had antitumor activity in patients with HER-positive gastric or EGJ cancer but provided limited survival benefit to patients with low HER2 expression [17]. In two Phase III clinical trials, ramucirumab, a monoclonal antibody VEGFR2 antagonist, was effective only in patients with previously treated advanced or metastatic gastric or EGJ cancer $[18,19]$. Most patients in these trials to investigate targeted agents had gastric adenocarcinoma; only 17-26\% had EGJ cancer [1719]. Current guidelines recommend pembrolizumab for second-line or subsequent therapy for tumors with high microsatellite instability or deficient mismatch repair and for third-line or subsequent therapy for PD-L1-positive esophageal and EGJ adenocarcinoma [8]. Less evidence is available for esophageal SCC than for adenocarcinoma; platinum-containing chemotherapy provides response rates of $20-50 \%$ and median overall survival duration of $8-10$ months [20,21]. Targeted agents have also been investigated as first-line therapy in patients with esophageal SCC, but these earlier attempts failed to demonstrate a clear benefit over chemotherapy alone $[10,21,22]$. Hence, there remains a significant need for novel therapeutic agents for the first-line treatment of advanced or metastatic esophageal or EGJ cancer.

\section{KEYNOTE-590 trial}

Herein, we describe the design and rationale for the randomized, double-blind, placebo-controlled Phase III KEYNOTE-590 study (ClinicalTrials.gov: NCT03189719), which will be conducted to evaluate the efficacy and safety of pembrolizumab plus chemotherapy compared with placebo plus chemotherapy as first-line treatment of patients with advanced esophageal or EGJ carcinoma.

\section{Background \& rationale}

Pembrolizumab is a high-affinity, highly selective, humanized immunoglobulin G4- $\kappa$ monoclonal antibody designed to block the interaction between PD-1 and its ligands, PD-L1 and PD-L2 [23,24]. Pembrolizumab has demonstrated robust, durable antitumor activity and a manageable safety profile against several advanced solid tumors and is approved in $>60$ countries for the treatment of one or more advanced cancers. The antitumor activity and safety of pembrolizumab in patients with esophageal carcinoma was previously investigated in the Phase Ib KEYNOTE-028 trial [25], the Phase II KEYNOTE-059 trial [26] and the Phase II KEYNOTE-180 trial [27].

The KEYNOTE-028 study included 23 patients with heavily pretreated, PD-L1-positive advanced esophageal cancer (78\% SCC, $22 \%$ adenocarcinoma) who received pembrolizumab $10 \mathrm{mg} / \mathrm{kg}$ every 2 weeks for up to 2 years. After a median follow-up of 7 months, the objective response rate (ORR) was 30\% (95\% CI: 13-53\%) with seven patients achieving confirmed partial response. ORR by histologic subtype was $28 \%$ (5/18 patients) for patients with SCC and 40\% (2/5 patients) for those with adenocarcinoma. The median time to initial response was 4 months (range: 2-8 months), and the median duration of response was 15 months (range: 6 to $\geq 26$ months) [25]. The KEYNOTE-059 study included 259 patients with previously treated gastroesophageal junction $(51 \%)$ or gastric (49\%) cancer who received pembrolizumab $200 \mathrm{mg}$ intravenously every 3 weeks until disease progression. After a median follow-up of 5.8 months, the ORR was 11.6\% (95\% CI: 8-16\%), with six $(2.3 \%)$ patients achieving complete response; the median duration of response was 8.4 months (range: $\geq 1.6$ to $\geq 17.3$ months) [26]. The KEYNOTE-180 (ClinicalTrials.gov: NCT02559687) trial is an open-label, Phase II study to evaluate pembrolizumab monotherapy as third-line therapy in patients with advanced adenocarcinoma or SCC of the esophagus or advanced Siewert Type 1 adenocarcinoma of the EGJ. Patients in the current trial were treated with pembrolizumab $200 \mathrm{mg}$ every 3 weeks for up to 2 years. After a median follow-up of 5.8 months, the ORR was 10\% (95\% CI: 5-17\%), with 12 patients achieving confirmed partial response. In patients with PD-L1-positive 
tumors, the ORR was 14\% (95\% CI: 6-25\%), whereas in patients whose tumors were PD-L1-negative, the ORR was 6\% (95\% CI: 2-16\%) [27].

In addition to the KEYNOTE-590 and KEYNOTE-180 studies, another clinical trial is underway to investigate the efficacy and safety of pembrolizumab in esophageal cancer. KEYNOTE-181 (ClinicalTrials.gov: NCT02564263) is a randomized, open-label, Phase III study to evaluate pembrolizumab monotherapy compared with physician's choice of standard therapy with paclitaxel, docetaxel or irinotecan in patients with advanced adenocarcinoma or SCC of the esophagus or advanced Siewert Type 1 adenocarcinoma of the EGJ that progressed after standard first-line therapy. The KEYNOTE-181 study has recently completed recruitment and is ongoing.

The promising antitumor activity demonstrated in the KEYNOTE-028 study in patients with heavily pretreated advanced esophageal cancer with both histologies and in the KEYNOTE-059 study in patients with pretreated gastroesophageal junction cancer and the continued unmet need for an effective treatment option for first-line advanced esophageal cancer support the early adoption of pembrolizumab as recommended third-line or subsequent therapy for PD-L1-positive esophageal and EGJ adenocarcinoma in recent US guidelines [28] and the investigation of pembrolizumab in patients with previously untreated advanced esophageal cancer. Furthermore, the addition of pembrolizumab to standard chemotherapy may translate to superior effectiveness and improved patient outcomes. It is known that platinum salts can have an iatrogenic impact on cancer evolution via the generation of neoantigens when tumor cells are destroyed [29], and, in esophageal cancers, these may be added to de novo mutations caused by common carcinogens, such as tobacco smoke and alcohol toxins [30]. Because the mutational landscape can determine sensitivity to PD-1 blockade, the presence of these neoantigens may increase pembrolizumab effectiveness and influence tumor regression $[31,32]$. Finally, in light of recent molecular classification data published by The Cancer Genome Atlas describing heterogeneous molecular subclasses [33], it is clear that biomarker analyses may provide information to assist in selecting those patients most likely to benefit from immunotherapy.

\section{Study design}

The KEYNOTE-590 study is a randomized, double-blind, placebo-controlled Phase III study (Figure 1). Eligible patients will be randomly assigned in a 1:1 ratio to receive pembrolizumab $200 \mathrm{mg}$ or placebo (normal saline) by intravenous (iv.) infusion every 3 weeks (Q3W) in combination with chemotherapy. The chemotherapy regimen for both arms will consist of cisplatin $80 \mathrm{mg} / \mathrm{m}^{2}$ iv. Q3W (maximum six doses) plus 5-fluorouracil $800 \mathrm{mg} / \mathrm{m}^{2}$ continuous iv. infusion on days 1-5 Q3W. Treatment will continue until confirmed radiographic progression, unacceptable toxicity, investigator or patient decision to withdraw, nonadherence to treatment or trial procedures or completion of 35 cycles of pembrolizumab or placebo (approximately 2 years).

Randomization will be performed using an interactive voice/web response system and will be stratified according to geographic region (Asia vs rest of world), histology (adenocarcinoma vs SCC), and Eastern Cooperative Oncology Group (ECOG) performance status (0 or 1). Pembrolizumab or placebo assignment will be masked to patients and investigators.

\section{Eligibility criteria}

Eligibility criteria are described in Table 1. Briefly, men and women aged 18 years or older were eligible for enrollment if they had a histologically or cytologically confirmed diagnosis of locally advanced unresectable or metastatic adenocarcinoma or SCC of the esophagus or if they had advanced/metastatic Siewert Type 1 adenocarcinoma of the EGJ.

\section{Planned sample size \& study period}

The planned sample size is approximately 700 patients. The study started in July 2017 , and the estimated study completion date is 22 August 2021.

\section{Outcome measures/end points}

Primary end points are progression-free survival (PFS) and overall survival (OS) in all patients and in the subgroup of patients with a PD-L1 combined positive score (CPS) $\geq 10$. PFS is defined as the time from randomization to first documented disease progression per Response Evaluation Criteria in Solid Tumors version 1.1 (RECIST v1.1) by blinded independent central review or death from any cause, whichever occurs first. OS is defined as the time from randomization to death from any cause. Secondary end points are safety and tolerability, ORR and duration of response per RECIST v1.1 by blinded independent central review and health-related quality of life 


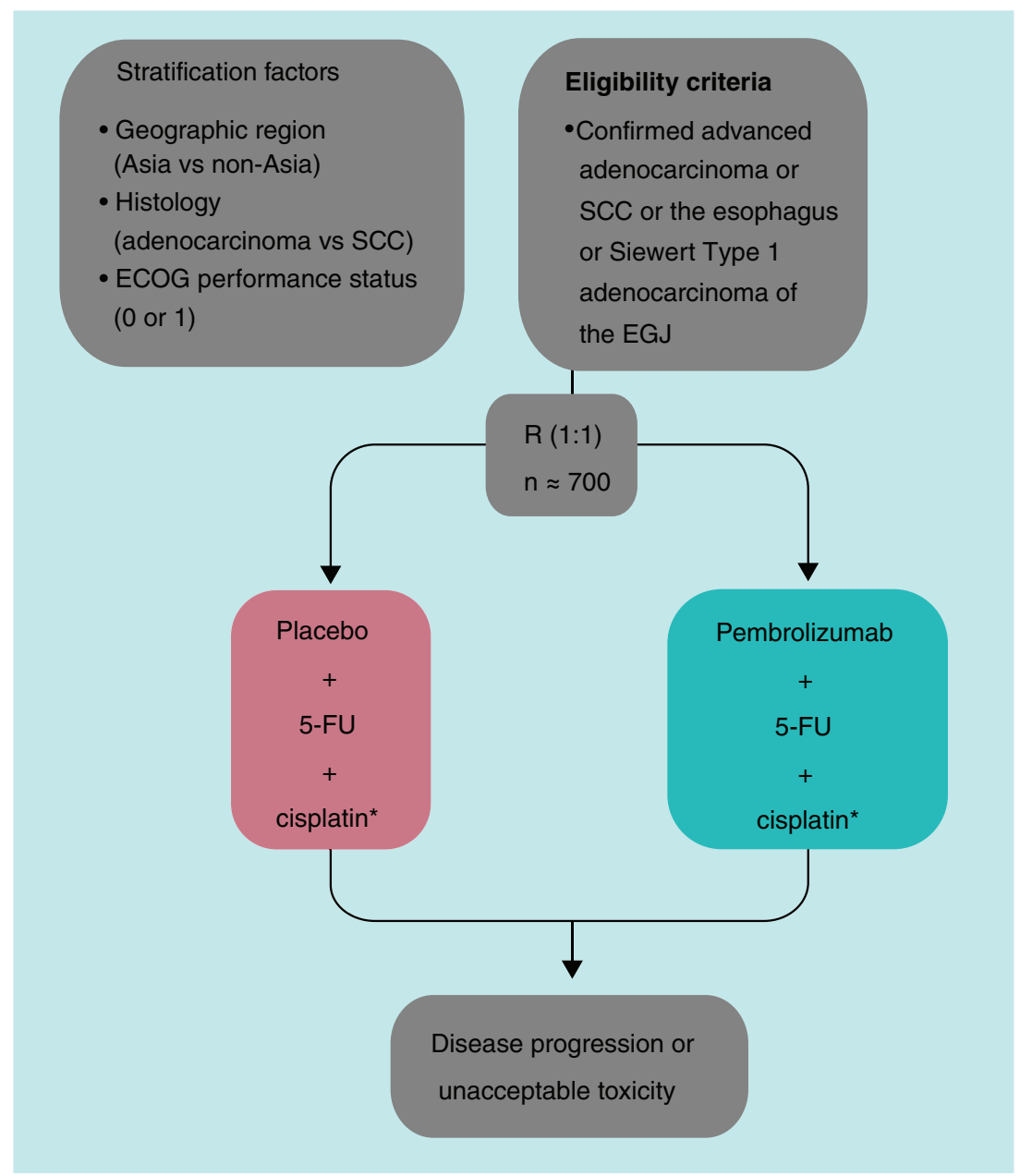

Figure 1. KEYNOTE-590 study design.

*Duration of cisplatin treatment will be capped at six cycles; however, treatment with 5-FU can continue per local standard.

5-FU: 5-fluorouracil; ECOG: Eastern Cooperative Oncology Group; EGJ: Esophagogastric junction; R: Randomization; SCC: Squamous cell carcinoma.

(assessed using the European Organization for the Research and Treatment of Cancer Quality of Life Questionnaire [EORTC QLQ] core 30 items [C30] and esophageal module [OES18]) in all patients and in patients with PD-L1 CPS $\geq 10$. Exploratory end points include characterization of utilities using the EuroQol 5D 5-level (EQ-5D-5L) questionnaire and the evaluation of PFS per immune-related RECIST by blinded independent central review in all patients and in patients with PD-L1 CPS $\geq 10$. Additional biomarkers that may be indicative of clinical response or resistance, safety, pharmacodynamic activity or mechanism of action of pembrolizumab may also be investigated.

\section{Study procedures}

Tumor response and disease progression will be assessed using computed tomography or magnetic resonance imaging if computed tomography is contraindicated. Initial tumor imaging will be performed during screening (within 21 days before randomization). Thereafter, imaging will be performed every 9 weeks (or more often if clinically indicated) until disease progression (confirmed by subsequent imaging $\geq 4$ weeks after first documentation of disease progression and verified by central review), start of new anticancer treatment, withdrawal of consent 
Table 1. Eligibility criteria for KEYNOTE-590.

\section{Inclusion criteria}

- Male or female

- Age $\geq 18$ years

- Histologically or cytologically confirmed locally advanced unresectable or metastatic adenocarcinoma or SCC of the esophagus or advanced Siewert Type 1 adenocarcinoma of the esophagogastric junction

- Measurable disease per RECIST v1.1 assessed by the local investigator

- ECOG performance status 0 or 1

- Provide newly obtained (preferred) or archival tissue sample

- Negative urine or serum pregnancy test within $72 \mathrm{~h}$ before randomization (females)

- Willing to use an adequate method of contraception throughout the study and for 120 days after the last dose of study medication and up to 180 days after the last dose of cisplatin

- Adequate hematologic function, defined as ANC $\geq 1500 / \mu \mathrm{l}$, platelet count $\geq 100,000 / \mu \mathrm{l}$ and hemoglobin $\geq 9.0 \mathrm{~g} / \mathrm{dl}$ or $\geq 5.6 \mathrm{mmol} / \mathrm{l}$

- Adequate renal function, defined as creatinine $\leq 1.5 \times$ ULN or measured or calculated creatinine clearance $\geq 60 \mathrm{~mL} / \mathrm{min}$ for those with creatinine levels $>1.5 \times$ ULN

- Adequate hepatic function, defined as total bilirubin

$\leq 1.5 \times$ ULN, or direct bilirubin $\leq$ ULN for those with total bilirubin

levels $>1.5 \times$ ULN, and ALT/AST levels $\leq 2.5 \times$ ULN

- Adequate coagulation function, defined as INR $\leq 1.5 \times$ ULN

unless the patient is receiving anticoagulant therapy as long as PT

or aPTT is within the therapeutic range

- Written informed consent

\section{Exclusion criteria}

- Locally advanced esophageal carcinoma that is resectable or potentially curable with radiation therapy per local investigator

- Previous therapy for advanced disease

- Major surgery, open biopsy or significant traumatic injury within 28 days before randomization or anticipated need for major surgery during the study treatment period

- Known additional malignancy that is progressing or requires active treatment (except for BCC or SCC of the skin, in situ cervical cancer, in situ breast cancer that has undergone potentially curative treatment and in situ or intramucosal pharyngeal cancer)

- Known active CNS metastases and/or carcinomatous meningitis; patients with previously treated and radiologically stable brain metastases may be eligible - Active autoimmune disease that has necessitated systemic treatment (other than replacement therapy) in the past 2 years

- Diagnosis of immunodeficiency, receiving chronic systemic steroid therapy $>10 \mathrm{mg}$ daily prednisone equivalent or any other form of immunosuppressive therapy within 7 days before the first dose of study treatment or history of organ transplant including allogeneic stem cell transplant

- Active infection necessitating systemic therapy

- History or current evidence of any condition, therapy or laboratory abnormality that might confound the study results or interfere with study participation - Known psychiatric or substance abuse disorder that would interfere with cooperation with study requirements

- Pregnant or breastfeeding or expecting to conceive within the projected study duration

- Prior treatment with anti-PD-1, anti-PD-L1 or anti-PD-L2 agent or an agent directed to another stimulatory or coinhibitory T-cell receptor (e.g., CTLA4, OX40 CD137)

- Known hypersensitivity (grade $\geq 3$ ) to any of the study drugs or their excipients - Known history of HIV, HBV or HCV infection

- Known history of active tuberculosis

- Prior radiotherapy within 14 days of randomization

- Receipt of live vaccine within 30 days before the first dose of study treatment

- Participation in a study of an investigational agent or device within 4 weeks before the first dose of study treatment

ALT: Alanine aminotransferase; ANC: Absolute neutrophil count; aPTT: Activated partial thromboplastin time; AST: Aspartate aminotransferase; BCC: Basal cell carcinoma; ECOG: Eastern Cooperative Oncology Group; HBV: Hepatitis B virus; HCV: Hepatitis C virus; INR: International normalized ratio; PT: Prothrombin time; SCC: Squamous cell carcinoma; ULN: Upper limit of normal.

or death, whichever occurs first. Patients will be followed up for survival status by telephone approximately every 12 weeks until death, withdrawal of consent or the end of the study (whichever occurs first).

Safety will be monitored throughout the study and for 30 days after the end of treatment (90 days for serious adverse events). Safety analysis will include the incidence, causality and outcome of adverse events; changes in vital signs; and changes in laboratory values. Adverse events will be graded and recorded throughout the trial and follow-up period per the National Cancer Institute Common Terminology Criteria for Adverse Events version 4.0.

Patient-reported outcome assessments (EORTC QLQ-C30, EORTC QLQ-OES18 and EQ-5D-5L) will be administered electronically on day 1 (before drug administration, adverse event evaluation and disease status notification) of each cycle during cycles 1-9, every three cycles thereafter for up to 1 year, at the time of treatment discontinuation and at 30 days after treatment discontinuation. Tumor PD-L1 expression will be assessed by use of a Good Manufacturing Practice immunohistochemistry assay (PD-L1 IHC 22C3 pharmDx; Agilent Technologies, CA, USA). PD-L1 is measured as the CPS, or the number of PD-L1-positive cells (tumor cells, lymphocytes and macrophages) divided by the total number of tumor cells, multiplied by 100 . Exploratory biomarker investigation may include genetic, RNA and proteomics analyses of blood and tumor samples.

\section{Statistics}

Efficacy will be assessed in the intent-to-treat population (all randomly assigned patients) and analyzed by randomized treatment group. Safety will be assessed in all randomly assigned patients who received at least one dose of study drug and will be analyzed by treatment received. The primary hypothesis for PFS and OS will be evaluated by comparing pembrolizumab plus chemotherapy with placebo plus chemotherapy using a stratified log-rank test. The study will be considered to have met its primary objective if at least one of the primary hypotheses is significant after controlling for multiplicity. Hazard ratios will be estimated using a stratified Cox proportional hazards regression 
model using the same factors for randomization (geographic region, histology and ECOG performance status). Event rates over time will be estimated using the Kaplan-Meier method.

Interim analyses are planned. An external data monitoring committee will periodically review efficacy and safety results to determine whether the study will continue per prespecified criteria.

\section{Conclusion}

Pembrolizumab demonstrated promising antitumor activity in the esophageal cohort of the Phase Ib KEYNOTE028 trial, which provided support for the further investigation of pembrolizumab in patients with advanced esophageal cancer. Preliminary data from the ongoing KEYNOTE-180 trial have also demonstrated promising antitumor activity in patients with previously treated, advanced/metastatic adenocarcinoma SCC of the esophagus or Siewert Type 1 adenocarcinoma of the gastroesophageal junction. In addition, the KEYNOTE-181 study is underway to investigate the safety and efficacy of pembrolizumab for previously treated advanced esophageal cancer. Herein, we described the methodology for the KEYNOTE-590 study, an ongoing Phase III study being conducted to investigate the safety and efficacy of pembrolizumab as first-line treatment for advanced esophageal carcinoma. The study aims to show that the combination of pembrolizumab with chemotherapy will provide improved effectiveness over chemotherapy alone and that the results from the KEYNOTE-590 study will help define the role of immunotherapy in patients with esophageal cancer, a patient population for whom treatment options are limited. In addition, biomarker data from this study may assist in determining the factors that influence the efficacy of immunotherapy and in selecting the patient subpopulations most likely to benefit from treatment with pembrolizumab.

\section{Summary points}

\section{Introduction}

- Treatment options for patients with unresectable advanced or metastatic esophageal or esophagogastric junction (EGJ) cancer are limited.

- Current guidelines for first-line treatment recommend chemotherapy containing a platinum and a fluoropyrimidine agent.

Background \& rationale

- In the Phase Ib KEYNOTE-028 trial in patients with heavily pretreated, PD-L1-positive advanced esophageal carcinoma, pembrolizumab demonstrated promising antitumor activity (objective response rate: $30 \%$ ) and a manageable safety profile.

- Pembrolizumab also demonstrated promising activity in the Phase II KEYNOTE-059 trial in patients with previously treated gastric or gastroesophageal junction cancer, thus supporting inclusion of pembrolizumab for third-line or subsequent therapy for PD-L1-positive esophageal and EGJ adenocarcinoma in the National Comprehensive Cancer Network Guidelines.

- Combining chemotherapy with pembrolizumab as first-line therapy may be beneficial for patients with advanced esophageal or EGJ cancer.

KEYNOTE-590 study design \& eligibility criteria

- KEYNOTE-590 is a randomized, double-blind, placebo-controlled, Phase III study to evaluate the efficacy and safety of pembrolizumab plus chemotherapy compared with placebo plus chemotherapy as first-line treatment for patients with advanced esophageal or EGJ cancer.

- Approximately 700 patients with previously untreated, locally advanced, unresectable or metastatic adenocarcinoma or squamous cell carcinoma of the esophagus or advanced or metastatic Siewert Type 1 adenocarcinoma of the EGJ will be enrolled.

- Eligible patients will be randomly assigned to receive pembrolizumab or placebo in combination with chemotherapy (cisplatin plus 5-fluorouracil).

Outcome measures/end points

- The primary end points are progression-free survival and overall survival in all patients and in patients who have a PD-L1 combined positive score $\geq 10$.

Conclusion

- It is hoped that results from the KEYNOTE-590 trial will help define the role of immunotherapy in patients with esophageal cancer. 


\section{Supplementary data}

An infographic accompanies this paper at the end of the references section. To download the infographic that accompanies this paper, please visit the journal website at: www.futuremedicine.com/doi/full/10.2217/fon-2018-0609

Author contributions

All authors were involved in the conception, design or planning of the study and critically reviewed and revised the manuscript for important intellectual content.

\section{Acknowledgements}

The authors thank the patients and their families and caregivers for participating in the study. Medical writing and/or editorial assistance was provided by J Walker and S Shaevitz of the ApotheCom pembrolizumab team (PA, USA).

\section{Financial \& competing interests disclosure}

This assistance was funded by Merck Sharp \& Dohme Corp., a subsidiary of Merck \& Co., Inc., NJ, USA. Funding for this research was provided by Merck Sharp \& Dohme Corp., a subsidiary of Merck \& Co., Inc., NJ, USA. K Kato has received research funding from Merck Sharp \& Dohme Corp., Merck Serono, ONO Pharmaceutical Co. Ltd and Shionogi, Inc. MA Shah has received research funding from Boston Biomedical, Inc., F Hoffmann-La Roche AG and Merck Sharp \& Dohme Corp. P Enzinger has acted as an advisor for Astellas Pharma, Inc., BeiGene Co, Celgene Corp., Eli Lilly and Co., Five Prime Therapeutics, Inc. and Merck Sharp \& Dohme Corp. J Bennouna has received honoraria from and acted as an advisor for AstraZeneca Plc, Boehringer-Ingelheim GmbH, F Hoffmann-La Roche AG, Merck Sharp \& Dohme Corp. and Shire Plc. A Adenis has received honoraria from Bayer AG, Bristol-Myers Squibb and Sanofi SA; acted as an advisor for Bayer AG, Bristol-Myers Squibb and Servier Laboratories; received research funding from Bayer AG, Bristol-Myers Squibb, Merck Sharp \& Dohme Corp., Pfizer, Inc. and Sanofi SA; and received travel, accommodation or expenses from Bayer AG, Bristol-Myers Squibb and Merck Sharp \& Dohme Corp. JM Sun has acted as an advisor for Boehringer-Ingelheim $\mathrm{GmbH}$ and received research funding from AstraZeneca Plc. BC Cho has received honoraria and acted as an advisor for AstraZeneca Plc, Boehringer-Ingelheim GmbH, Bristol-Myers Squibb, F Hoffmann-La Roche AG, Merck Sharp \& Dohme Corp., Novartis International AG and Yuhan Co. Ltd; served on speaker bureaus for AstraZeneca Plc, Bristol-Myers Squibb, Merck Sharp \& Dohme Corp. and Novartis International AG; and received research funding from AstraZeneca Plc, Bayer AG, Novartis International AG and Yuhan Co. Ltd. M Ozguroglu has received honoraria from Janssen Pharmaceutica NV and acted as an advisor for Astellas Pharma, Inc. and Janssen Pharmaceutica NV. T Kojima has received honoraria from Oncolys Biopharma, Inc. and has intellectual property interests in Amgen, Inc., Astellas Pharma, Inc., Merck Sharp \& Dohme Corp., Oncolys Biopharma, Inc., ONO Pharmaceutical Co. Ltd and Shionogi, Inc. Y Zhu, LA McLean and S Shah are employees of Merck Sharp \& Dohme Corp., a subsidiary of Merck \& Co., Inc., NJ, USA. LA McLean and S Shah own stock in the company. T Doi has acted as an advisor for Amgen Inc., Chugai Pharmaceutical Co. Ltd, Daiichi Sankyo Co. Ltd, Kyowa Hakko Kirin Co. Ltd, Eli Lilly Japan KK, Merck Sharp \& Dohme Corp. and Novartis International AG; and received research funding from Astellas Pharma, Inc., Bayer AG, Boehringer Ingelheim GmbH, Celgene Corp., Chugai Pharmaceutical Co. Ltd, Daiichi Sankyo Co. Ltd, Janssen Pharmaceutica NV, Kyowa Hakko Kirin Co. Ltd, Eli Lilly Japan KK, Merck Serono, Merck Sharp \& Dohme Corp., Novartis International AG, Pfizer, Inc., Sumitomo Corp., Taiho Pharmaceutical Co. Ltd and Takeda Pharmaceutical Co. Ltd. C Hierro has received research funding from Bayer and lecture fees and travel grants from Lilly, Ignyta and Roche. L Shen and V Kostorov report no conflicts of interest. The authors have no other relevant affiliations or financial involvement with any organization or entity with a financial interest in or financial conflict with the subject matter or materials discussed in the manuscript apart from those disclosed.

\section{Ethical conduct of research}

The authors state that they obtained appropriate institutional review board approval and followed the principles outlined in the Declaration of Helsinki for all human experimental investigations and that they received informed consent from the participants involved.

\section{References}

Papers of special note have been highlighted as: $\bullet \bullet$ of considerable interest

1. WHO. GLOBOCAN 2012 estimated cancer incidence, mortality and prevalence worldwide. http://globocan.iarc.fr/Default.aspx

2. Hassanipour S, Mohammadian-Hafshejani A, Ghoncheh M, Salehiniya H. The incidence and mortality of esophageal cancer and its relationship with development in the world. Biomed. Res. Ther. 4(9), 1607-1623 (2017).

3. Siegel RL, Miller KD, Jemal A. Cancer statistics, 2018. CA Cancer J. Clin. 68(1), 7-30 (2018). 
4. National Cancer Institute SEER. Previous version: SEER cancer statistics review, 1975-2011. https://seer.cancer.gov/archive/csr/1975_2011/

5. Pennathur A, Gibson MK, Jobe BA, Luketich JD. Oesophageal carcinoma. Lancet 381(9864), 400-412 (2013).

6. Thrift AP, Whiteman DC. The incidence of esophageal adenocarcinoma continues to rise: analysis of period and birth cohort effects on recent trends. Ann. Oncol. 23(12), 3155-3162 (2012).

7. Thrift AP. The epidemic of oesophageal carcinoma: where are we now? Cancer Epidemiol. 41, 88-95 (2016).

8. National Comprehensive Cancer Network. NCCN clincial practice guidelines in oncology. Esophageal and esophagogastric junction cancers. Version 2.2018. www.nccn.org/professionals/physician_gls/default.aspx

9. Lordick F, Mariette C, Haustermans K, Obermannova R, Arnold D. Oesophageal cancer: ESMO clinical practice guidelines for diagnosis, treatment and follow-up. Ann. Oncol. 27(Suppl. 5), v50-v57 (2016).

10. Lorenzen S, Schuster T, Porschen R et al. Cetuximab plus cisplatin-5-fluorouracil versus cisplatin-5-fluorouracil alone in first-line metastatic squamous cell carcinoma of the esophagus: a randomized Phase II study of the Arbeitsgemeinschaft Internistische Onkologie. Ann. Oncol. 20(10), 1667-1673 (2009).

11. Thuss-Patience PC, Kretzschmar A, Bichev D et al. Survival advantage for irinotecan versus best supportive care as second-line chemotherapy in gastric cancer: a randomised Phase III study of the Arbeitsgemeinschaft Internistische Onkologie (AIO). Eur. J. Cancer 47(15), 2306-2314 (2011).

12. Ford HE, Marshall A, Bridgewater JA et al. Docetaxel versus active symptom control for refractory oesophagogastric adenocarcinoma (COUGAR-02): an open-label, Phase III randomised controlled trial. Lancet Oncol. 15(1), 78-86 (2014).

13. Janowitz T, Thuss-Patience P, Marshall A et al. Chemotherapy vs supportive care alone for relapsed gastric, gastroesophageal junction, and oesophageal adenocarcinoma: a meta-analysis of patient-level data. Br. J. Cancer 114(4), 381-387 (2016).

14. Kang JH, Lee SI, Lim DH et al. Salvage chemotherapy for pretreated gastric cancer: a randomized Phase III trial comparing chemotherapy plus best supportive care with best supportive care alone. J. Clin. Oncol. 30(13), 1513-1518 (2012).

15. Hironaka S, Ueda S, Yasui H et al. Randomized, open-label, Phase III study comparing irinotecan with paclitaxel in patients with advanced gastric cancer without severe peritoneal metastasis after failure of prior combination chemotherapy using fluoropyrimidine plus platinum: WJOG 4007 trial. J. Clin. Oncol. 31(35), 4438-4444 (2013).

16. Kato K, Tahara M, Hironaka S et al. A Phase II study of paclitaxel by weekly 1-h infusion for advanced or recurrent esophageal cancer in patients who had previously received platinum-based chemotherapy. Cancer Chemother. Pharmacol. 67(6), 1265-1272 (2011).

17. Bang YJ, Van Cutsem E, Feyereislova A et al. Trastuzumab in combination with chemotherapy versus chemotherapy alone for treatment of HER2-positive advanced gastric or gastro-oesophageal junction cancer (ToGA): a Phase III, open-label, randomised controlled trial. Lancet 376(9742), 687-697 (2010).

18. Fuchs CS, Tomasek J, Yong CJ et al. Ramucirumab monotherapy for previously treated advanced gastric or gastro-oesophageal junction adenocarcinoma (REGARD): an international, randomised, multicentre, placebo-controlled, Phase III trial. Lancet 383(9911), 31-39 (2014).

19. Wilke H, Muro K, Van Cutsem E et al. Ramucirumab plus paclitaxel versus placebo plus paclitaxel in patients with previously treated advanced gastric or gastro-oesophageal junction adenocarcinoma (RAINBOW): a double-blind, randomised Phase III trial. Lancet Oncol. 15(11), 1224-1235 (2014).

20. Kato K, Muro K, Ando N et al. A Phase II study of nedaplatin and 5-fluorouracil in metastatic squamous cell carcinoma of the esophagus: The Japan Clinical Oncology Group (JCOG) Trial (JCOG 9905-DI). Esophagus 11(3), 183-188 (2014).

21. Adenis A, Bennouna J, Etienne P-L et al. Discontinuation of first-line chemotherapy (CT) after 6weeks of CT in patients (pts) with metastatic squamous-cell esophageal cancer (MSEC): a randomized Phase II trial. J. Clin. Oncol. 34(Suppl. 15), 4002 (2016).

-• Discontinuation Phase II study assessing survival outcomes in patients with metastatic squamous cell esophageal cancer who were free from progression after 6-week treatment with first-line 5-fluorouracil/platinum-based chemotherapy.

22. Moehler MH, Thuss-Patience P, Brenner B et al. Cisplatin/5-FU (CF) +/- panitumumab (P) for patients (pts) with non-resectable, advanced, or metastatic esophageal squamous cell cancer (ESCC): an open-label, randomized AIO/TTD/BDGO/EORTC Phase III trial (POWER). J. Clin. Oncol. 35(Suppl. 15), 4011 (2017).

-• Multicenter, open-label, randomized, Phase III study (POWER) reporting the safety and efficacy of cisplatin/5-fluorouracil with or without panitumumab in patients with nonresectable, advanced or metastatic esophageal squamous cell cancer.

23. Merck Sharpe \& Dohme Corp., KEYTRUDA ${ }^{\circledR}$ (pembrolizumab) injection, for intravenous use. NJ, USA, (2018). www.merck.com/product/usa/pi_circulars/k/keytruda/keytruda_pi.pdf

24. Mcdermott J, Jimeno A. Pembrolizumab: PD-1 inhibition as a therapeutic strategy in cancer. Drugs Today 51(1), 7-20 (2015).

25. Doi T, Piha-Paul SA, Jalal SI et al. Safety and antitumor activity of the anti-programmed death-1 antibody pembrolizumab in patients with advanced esophageal carcinoma. J. Clin. Oncol. 36(1), 61-67 (2018).

-• Multicenter, multicohort, Phase Ib study (KEYNOTE-028) reporting the safety, efficacy and gene expression signature score of a cohort of patients with recurrent or metastatic PD-L1-positive esophageal or gastroesophageal junction cancer who were treated with pembrolizumab. 
26. Fuchs CS, Doi T, Jang RW et al. Safety and efficacy of pembrolizumab monotherapy in patients with previously treated advanced gastric and gastroesophageal junction cancer: Phase II clinical KEYNOTE-059 trial. JAMA Oncol. 4(5), e180013 (2018).

-• Global, single-arm, multicohort, Phase II study (KEYNOTE-059) reporting the safety and efficacy of pembrolizumab monotherapy in a cohort of patients with previously treated, advanced gastric or gastroesophageal junction cancer.

27. Shah MA, Kojima T, Hochhauser D et al. Efficacy and safety of pembrolizumab for heavily pretreated patients with advanced, metastatic adenocarcinoma or squamous cell carcinoma of the esophagus: Phase II KEYNOTE-180 study. JAMA Oncol. doi:10.1001/jamaoncol.2018.5441 (2018).

-• Preliminary findings of the open-label, Phase II KEYNOTE-180 study evaluating the efficacy and safety of pembrolizumab in patients with previously treated, advanced or metastatic adenocarcinoma or squamous cell carcinoma of the esophagus or Siewert Type 1 adenocarcinoma of the gastroesophageal junction.

28. National Comprehensive Cancer Network. NCCN guideline. Esophageal and esophagogastric junction cancers. Version 4. 2017. www.nccn.org/professionals/physician_gls/default.aspx

29. Murugaesu N, Wilson GA, Birkbak NJ et al. Tracking the genomic evolution of esophageal adenocarcinoma through neoadjuvant chemotherapy. Cancer Discov. 5(8), 821-831 (2015).

30. Lawrence MS, Stojanov P, Polak P et al. Mutational heterogeneity in cancer and the search for new cancer-associated genes. Nature 499(7457), 214-218 (2013).

31. Rizvi NA, Hellmann MD, Snyder A et al. Mutational landscape determines sensitivity to PD-1 blockade in non-small cell lung cancer. Science 348(6230), 124-128 (2015).

32. Snyder A, Makarov V, Merghoub T et al. Genetic basis for clinical response to CTLA-4 blockade in melanoma. N Engl. J. Med. 371(23), 2189-2199 (2014).

33. The Cancer Genome Atlas Network. Integrated genomic characterization of oesophageal carcinoma. Nature 541(7636), 169-175 (2017). 


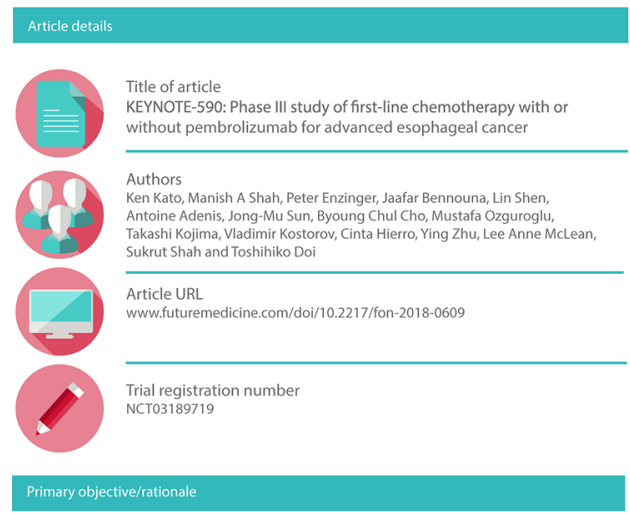

Primary objective

Compare between treatment arms PFS per RECIST 1.1, as determined by blinded CPS $\geq 10$

Secondary objectives

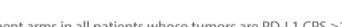
ORR per RECIST 1 1. as determined by blinded independent central review - DOR per RECIST 1.1, as determined by blinded independent central review Changes from baseline in HRQOL using the EORTC QLQ-C30 and the EORTC QLQ-OES18

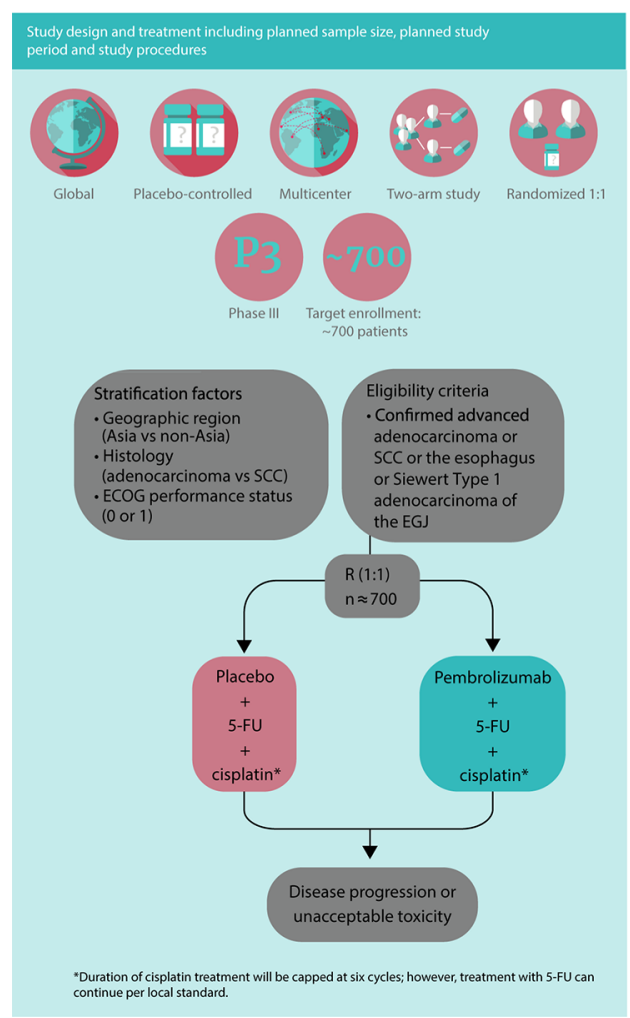

\section{Study design and treatment including planned sample size, planned study
period and study procedures (cont)}

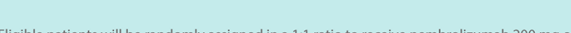
Eligible patients will be randomly assigned in a 1:1 ratio to receive pembrolizumab 200 chemotherapy regimen for both arms will consist of cisplatin $80 \mathrm{mg} / \mathrm{m}^{2} \mathrm{iv}$. Q3W (capped at 6 doses) plus $5-\mathrm{FU} 800 \mathrm{mg} / \mathrm{m}^{2}$ continuous iv. infusion on days $1-5$ Q3W. Treatment will contin until confirmed radiographic progression, unacceptable toxicity, investigator or patient cycles of pembroling nabor phace (-2years)

Randomized patients will be stratified according to geographic region (Asia vs rest of world), histology (adenocarcinoma vs SCC), and ECOG performance status ( 0 or 1 ). Pembrolizumab or placebo assignment wil be masked to patients and investigators.

The planned sample size is $\sim 700$ patients. The study started in July 2017 , and the estimated study completion date is August 22, 202

\section{Key eligibility criteria}

Age 218 years, male or female

Histologically or cytologically confirmed diagnosis of locally advanced unresectable or metastatic adenocarcinoma or sCC of the esophagu or
advanced/metastatic Siewert type 1 adenocarcinoma of the EGJ

Measurable disease per RECIST v1.1 assessed by the local investigator ECOG performance status 0 or

Provide newly obtained (preferred) or archival tissue sample

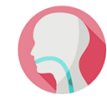

Negative urine or serum pregnancy test within $72 \mathrm{~h}$ before randomization (females)

Willing to use an adequate method of contraception throughout the study and for 120 days after the last dose of study medication and up to 180 days after the last dose of cisplatin

Adequate hematologic function, defined as ANC $\geq 1500 / \mu$, platelet count $\geq 100,000 / \mu l$ and hemoglobin $\geq 9.0 \mathrm{~g} / \mathrm{dl}$ or $\geq 5.6 \mathrm{mmol} / \mathrm{d}$

Adequate renal function, defined as creatinine $>1.5 \times$ UIN or measured or calculated creatinin clearance $\geq 60 \mathrm{ml} / \mathrm{min}$ for those with creatinine levels $>1.5 \times$ ULN

Adequate hepatic function, defined as total bilirubin $\leq 1.5 \times$ ULN or direct bilirubin $\leq U L N$ for those with total bilirubin levels $>1.5 \times$ ULN, and ALT/AST levels $\leq 2.5 \times$ ULN

Adeguate coagulation function, defined as INR $\leq 1.5 \times$ ULN unless the patient is receiving anticoagulant therapy as long as PT or aPT is within the therapeutic range

Written informed consent

\section{Outcome measures/end points}

Primary end points

PFS and OS in all patients and in the subgroup of patients with PD-LI CPS 210 . PFS is defined as the time from randomization to first documentreview or death from any cause, whichever occurs first OS is defined as the

Secondary end points

Safety and tolerability, ORR and duration of response per RECIST v1.? life (assessed using the EORTC QLQ C 30 and esophageal module) in al patients and in patients with PD-L1 CPS $\geq 10$

Exploratory end points

Utilities using EQ-5D-5L questionnaire, PFS per immune-related RECIST by blinded independent central review in all patients and in patients with PD-LI CPS $\geq 10$, molecular biomarkers that might be indicative of response, resistance, safety, pharmacodynamic activity or mechanism of

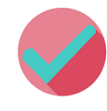

\section{Glossary}

ALT: Alanine aminotransferase; ANC: Absolute neutrophil count; aPTT: Activated partia thromboplastin time; AST: Aspartate aminotransferase; CPS: Combined positive score EG. Esoph

EORTC OPO Organisation for the Research and Treatment of Cancer; EQ-5D-5L: Euro Ool 5-dimension 5-level INR: International normalized ratio; ORR: Objective response rate; OS: Overall survival; PFS: Progression-free survival, Q3W: Every 3 weeks, RECIST V1.1. R: Response Evaluation Criteria in
Solid Tumors version 1.1: SCC. Squamous cell carcinoma: ULN: Upper limit of normal 